\title{
The Investigation of Drug Resistance Substitutions in NS3 Protease Sequence of Hepatitis C Virus from Non-Responder Patients
}

\author{
Nargess Nejabat ${ }^{1}$, Seyed Younes Hosseini ${ }^{1 *}$, Jamal Sarvari ${ }^{1}$, Ali Akbar Gorzin ${ }^{1}$, \\ Mohammad Reza Fattahi², Mohammad Rasoolian ${ }^{3}$
}

\begin{abstract}
Background: Even with the fantastic successes of direct-acting antivirals (DAA) in the treatment of Hepatitis C Virus (HCV) infection, natural drug resistance remains a challenging obstacle for their impacts. The data regarding protease inhibitors (PIs) resistance in Iran population are limited. The aim of this study was to investigate the variations in NS3 protease of HCV from non-responder patients. Methods: In this cross-sectional study, $14 \mathrm{HCV}$ infected patients with genotype $1(\mathrm{~N}=5)$ and $3(\mathrm{~N}=9)$ who have not responded to Interferon-related regime were enrolled from Liver Clinic, Shiraz. The NS3 protease region was amplified by Nested-PCR followed by product gel extraction. Besides, some amplified protease regions were cloned into a cloning vector to improve the sensitivity of mutation detection. Both crude and cloned sequences were then introduced into sequencing. The obtained sequences were compared with the NS3 reference sequences and analyzed by Geno2pheno available software to find possible substitutions. In the end, the phylogenetic tree was constructed. Results: Among variations responsible for PIs resistance, only one out of 14 (7\%) sample who was infected with genotype 1a, harbored $R 117 C+N 174 S$ double mutation, which causes reduced susceptibility to Telaprevir. Any another resistance mutation was not found among the studied population. The most frequent substitutions were determined as $I 52 M(N=9), S 102 A(N=9), S 166 A(8)$ and V170I(8) for genotype 3a, and $F 147 S / A(4)$ for genotype 1. However, some uncharacterized substitutions on scored position, including $I 132 L(N=1)$, $\operatorname{I170V}(\mathrm{N}=3)$ and $\mathrm{N174S}(\mathrm{N}=2)$ were also determined among sequences. Phylogenetic analysis demonstrated that the protease region has enough power to correctly classify enrolled samples into relevant clusters on the tree. There were 2 , 3 and 9 cases of sub-genotypes 1a, 1b, and 3a, respectively. Conclusion: A low frequency of PIs resistance mutations in our HCV infected population is a hopeful point of starting these drugs in HCV infected patients.
\end{abstract}

Keywords: Hepatitis C virus- protease inhibitors- NS3- phylogenetic analysis- drug resistance

Asian Pac J Cancer Prev, 20 (8), 2311-2317

\section{Introduction}

Hepatitis $\mathrm{C}$ virus (HCV) is a blood-borne hepatotropic member of the Flaviviridae that can establish end-stage liver diseases. It is estimated that more than 185 million people are persistently infected worldwide (Petruzziello et al., 2016). Regarding the full sequence analysis, HCV has been classified into at least 7 genotypes and a number of sub-genotypes (Khodabandehloo and Roshani, 2014).

Hepatitis $\mathrm{C}$ virus therapy has been facing a revolution following the discovery of direct-acting antiviral drugs (DAAs) that leads the highest rate of sustained virologic response ever (Paolucci et al., 2013). However, one of the main problems with anti-HCV DAAs generation is the presence of natural resistant variants which arise spontaneously (Jindal et al., 2017). These variations may lead to virological response failure during HCV treatment. Thus, an early evaluation of the DAAs resistance could be beneficial to prevent treatment failure and to improve the HCV therapy management.

Three classes of DAAs are candidates of marketing nowadays that among them telaprevir, boceprevir and glecaprevir are NS3 protease inhibitors (PIs) (Lamb, 2017; Morsica et al., 2017). PIs drugs inhibit NS3 protease activity by occupying the activation site or surrounding motifs, consequently inhibiting HCV viral replication (Lamb, 2017). NS3 protease is a very attractive target for HCV therapy by synthesized protease inhibitors (PIs), however natural appearance of variability in NS3 protease sequence influences its susceptibility to these drugs.

The enzymatic protease activity of NS3 protein mapped to 189 amino acids from its amino-termini (Courcambeck

${ }^{I}$ Department of Bacteriology and Virology, School of Medicine, ${ }^{2}$ Gastroenterohepatology Research Center, Shiraz University of Medical Sciences, Shiraz, ${ }^{3}$ Department of Genetics and Molcular Biology, School of Medicine, Isfahan University of Medical Sciences, Isfahan, Iran.*For Correspondence: hoseiniy@sums.ac.ir 
et al., 2006; Khanlari et al., 2014). This activity has postulated to be involved in some $\mathrm{HCV}$ pathogenesis such as fibrogenesis and immune suppression (Khanlari et al., 2014; Khanizadeh et al., 2016). The error-prone property of RNA-dependent RNA polymerase (RDRP) of $\mathrm{HCV}$, introduces some substitutions in virus sequence during replication (Courcambeck et al., 2006; Bartels et al., 2008). Actually, this kind of diversity induces the expansion of natural drug resistance strains before the start point of therapy(Bartels et al., 2008). The natural and pre-existing drug resistance substitutions in the protease region have been reported in a couple of reports (Bartels et al., 2008; Paolucci et al., 2012; Vicenti et al., 2012; Zeminian et al., 2013; Jindal et al., 2017). There are different well-defined substitutions alongside the protease region that are responsible for PIs resistant, including V36L, T54S, V55A, Q80H, R155K, A156S and V170A and $V 36 M$ which may affect the therapy response (Vallet et al., 2011; Jindal et al., 2017; Martinez et al., 2017).

In our country, there are limited studies that considered PIs mutations. Afrasiabi et al; interestingly found the critical $V 36 L$ and $R 155 \mathrm{~K}$ substitutions among a small population ( 7 patients) when analyzed by clonal sequencing (Afrasiabi et al., 2015). However, regarding this finding, a conclusion about the rate of natural PIs mutations in our patient population is far to be reached. Furthermore, screening of the natural substitutions of protease is critical based on their cost and effectiveness (Lopez-Labrador et al., 2008; Romano et al., 2010).

Therefore, the aim of this study was to investigate the sequence of the protease region of NS3 from $\mathrm{HCV}$-infected patients who did not respond to standard IFN+Ribavirin therapy. For this purpose, the $N S 3$ protease sequence of 14 non-responder patients to standard IFN+Ribavirin therapy was analyzed by simple sequencing method and to ensure the fidelity, some of them were also subjected to clonal-sequencing as well. This investigation revealed a low frequency of mutations related to PIs reduced sensitivity but not PIs resistance in samples.

\section{Materials and Methods}

\section{Patients}

A total of 14 confirmed chronic HCV patients that have not responded to standard Interferon+Ribavirine therapy (named as IFN/RIB Non-responder) or relapsed afterwards, were selected from the Liver Clinic of Shahid Motahari Clinic (Shiraz, Iran), in 2016. The IFN/RIB Non-responders were selected due to their priority for taking DAA medications such as protease inhibitors regarding the liver specialist advice. All patients had fulfilled a course of standard peg-interferon/ribavirin therapy, but no PIs drug consumption. Also, they were negative for HIV and HBV infection based on their medical records. To determine the sample size, we considered $\alpha$ and $\beta$ as 0.05 and $=0.2$, respectively. Also, the power of our test was PASS.

The Ethics Committee affiliated with Shiraz University of Medical Sciences has approved the study and written informed consent was obtained from all the participants before sampling. Blood samples were taken in tubes and serum parts were collected in sterile condition, and were stored at $-70^{\circ} \mathrm{C}$ until further tests.

\section{Primer Design}

At first, the reference HCV genotypes and subtypes (those with genotype $1 a, 1 b$, or $3 a$ ) full length sequences, were retrieved from NCBI (National Center for biotechnology Information) and the LOS Alamos database. Then, multiple sequence alignments were performed by Mega7 software. The primer sequences covering 203 amino acids from the protease region of NS3, were designed by Oligo 7 and primer3 software so that they can detect the sequences associated with genotype $1 a, 1 b$ and $3 a$. The primers are listed in Table 1 .

\section{HCV genome detection in serum samples}

Before starting the amplification of NS3 sequence of samples, the presence of $H C V$ genome was evaluated by an in-house nested PCR assay, as described before (Afrasiabi et al., 2015). It was performed by specific pairs of primers targeting 5/noncoding region, NCR. These primers were designed to amplify the early $5 / \mathrm{NCR}$ region of all $\mathrm{HCV}$ genotypes.

\section{Amplification of NS3 Protease Region}

Viral RNA was extracted from the serum samples using an RNA extraction kit (Stratec inc., Germany), according to the instruction. Then, complementary DNA (cDNA) synthesis and first amplification reaction were performed on viral RNA using the commercially available kit (Onestep cDNA synthesis Takara kit, Takara Inc,. Japan), and common primers targeting protease region of all sub-genotypes ( $N J$-Com $A 1$ and $N J-C o m A 2$ pair of primers) based on the recommended instructions. After this, a nested-PCR was performed with the help of one of specific $N J$-In a1, NJ-In b1, and NJ-F3a forward primers and a common $N J$-ComA2 reverse primer to amplify sequences from genotype $1 \mathrm{a}, 1 \mathrm{~b}$ and $3 \mathrm{a}$, respectively. Nested-PCR steps were including a primary denaturation at $94^{\circ} \mathrm{C}$ for 4 minutes, then, 35 cycles of denaturation at $94^{\circ} \mathrm{C}$ for 40 seconds, annealing at $51^{\circ} \mathrm{C}$ for 40 seconds and extension at $72^{\circ} \mathrm{C}$ for 45 seconds; In the end, the desired product sequences were purified from agarose gel, in accordance with the manual of the gel extraction kit (MACHERY-NAGEL(MN), Germany).

\section{Cloning and Sequencing}

In order to enhance the sensitivity of variation detection in NS3 sequence, some of them cloned into vector then introduced into sequencing. In this step, the purified PCR product was cloned using TA/cloning system kit (CinnaGen Inc., Iran) containing linear vector $p T G 19-T$. Following the kit instructions, ligation was performed using T4 ligase enzyme at $37^{\circ} \mathrm{C}$. Then, the reaction product was transformed into competent $\mathrm{DH} 5 \alpha$ bacteria. The bacteria were grown on LB agar, containing ampicillin (50 $\mu \mathrm{g} / \mathrm{mL}$ ) along with X-Gal and IPTG. Recombinant true plasmids were initially screened by blue/white screening. Purification of true recombinant plasmids was done using Plasmid DNA Purification kit, based on the recommended instruction (MACHEREY-NAGEL Company, Germany). 
For each patient, nearly 3 colonies of plasmids were sent for sequencing (Eurofins Genomics Company., Germany), which were performed bidirectional using $N J$-In al or $N J-I n$ b1 or $N J-F 3 a$ and $N J-C o m A 1$ primers set.

\section{Sequence variation Analysis and Phylogeny}

The NS3 sequence of different reference genotypes of HCV was retrieved from NCBI database, then, aligned using MEGA7 software. The employed reference sequences accession numbers for further analysis were as follows: genotype 1a, NC004102, D10749 and DQ068207; genotype 1b, AY460204, U01214, and AF176573; genotype $1 C, A Y 651061$ and D14853; genotype 2, AY746460 and AF169005; genotype 3a, D28917; genotype 4, Y11604; genotype 5, AF064490; and genotype 6, Y12083.

The sequencing results were retrieved through Chromas software, and were then multiple aligned by the help of Clustal W method, using Mega7 software. The variations responsible for PIs resistance were detected by comparing with reference sequences. In the other word, for substitution analysis, the major well-defined amino acid residues responsible for "complete PIs resistance", including V36, T54, V55, Q80, R155, A156, $D 168$ and $I / V 170$ as well as those attributed to "potential resistance" such as F43, Y56,V107, R117, S122 and S174 were considered (Sargin Altunok et al., 2016; Zhang et al., 2016). Hence, the sequencing results were also submitted to geno2pheno online analysis system to detect substitutions with PIs resistance importance. In this regard, substitutions categorized as "resistance" or "reduced sensitivity" mutations as described before (Kalaghatagi et al,. 2016). This system was also employed to confirm the genotypes of investigated samples as well.

Furthermore, as HCV samples were pre-defined in the case of genotype, they were investigated if those commercial genotyping assay could be compatible with phylogenetic analysis of protease sequence. For this purpose, Phylogenic tree was constructed by the neighbor-joining method using MEGA7 software. Finally, the created tree was evaluated by 500 times bootstrapping to ensure its fidelity.

\section{Results}

\section{Patients}

The mean age of subjects was $46.6 \pm 12.3$ years with a range of 29-66 years. The gender of 10 participants was men and 4 others were women.

\section{The virus infection screening}

In order to screen the virus genome presence, nested PCR was employed to target 5'NTR region. The test was positive for all enrolled serum samples. A band with near

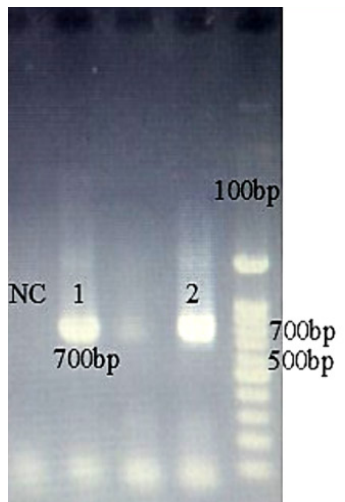

A

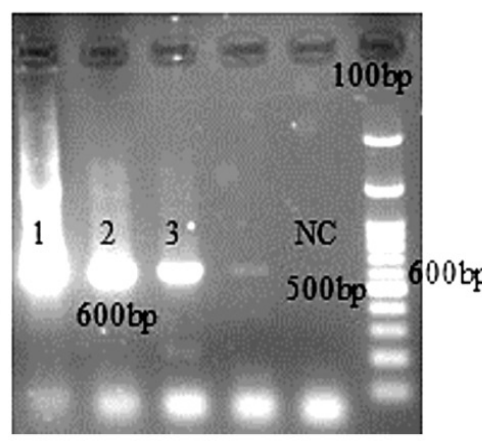

B

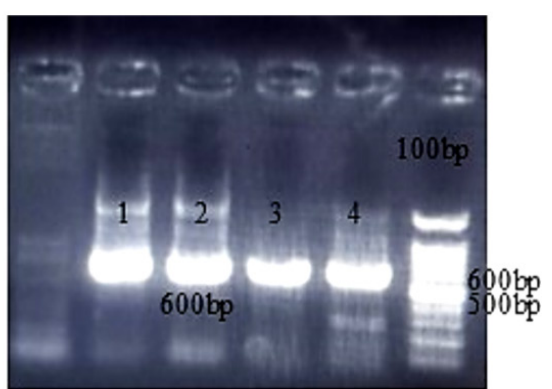

C

Figure 1. The Gel Electrophoresis Analysis of Different Nested-PCRs. A, The presence of a 700 bp band in samples 1 and 2 were indicative of amplification of samples with 1a sub-genotypes; B, The appearance of a $600 \mathrm{bp}$ band in line 1,2 and 3 were indicative of positivity of these samples for sub-genotype $1 \mathrm{~b} ; \mathrm{C}$, The presence of a $600 \mathrm{bp}$ band in line $1,2,3$, and 4 was a marker of sub-genotype 3a. The size marker was 100 bp ladder (Cinnaclone Inc.).

Table 1. Designed Primers for Amplification of 3 Subgenotypes 1a, 1b and 3a

\begin{tabular}{lll}
\hline Primer Name & \multicolumn{1}{c}{ *Sequence 5' - 3' } & \multicolumn{1}{c}{ Application } \\
\hline NJ-Com A1 (Forward) & ATCACVTGGGGVGCRGAYAC & First round amplification (all genotypes) \\
NJ-Com A2 (Reverse) & AACTTRCCRTAGGTGGARTABGT & First round amplification (all genotypes) \\
NJ-In a1 (Forward) & CGAYGGAATGGTCTCCAAG & Second amplification (specialized for genotype 1a) \\
NJ-In a2 (Reverse) & CRGCAACRGAGGGGTTGAG & Second amplification (specialized for genotype 1a) \\
NJ-In b1 (Forward) & GGCCTRCTTGGYTGCATCAT & Second amplification (specialized for genotype 1b) \\
NJ-In b2 (Reverse-1) & GTAGRTGGGCCACTTGAAAT & Second amplification (specialized for genotype 1b) \\
NJ-In b3 (Reverse-2) & TYAGGACGAGYACCTTGTA & Second amplification (specialized for genotype 1b) \\
NJ-F3a (Forward) & GGGCCTTYTTGGGACTATTG & Second amplification (specialized for genotype 3a). \\
NJ-R3a (Reverse) & GGGACCTTTGTGCTYTTRCC & Second amplification (specialized for genotype 3a). \\
\hline
\end{tabular}

*Degenerative Base key: R:A or G; Y: C or T; V: A, C or G; B:C, G or T 
Table 2. The Substitutions and Their Frequency in Sample Sequences. The more prevalent substitutions were indicated by underline marker and PIs resistance mutations were bolded

\begin{tabular}{ll}
\hline Genotype & \multicolumn{1}{c}{ Variations } \\
\hline 1a and 1b(N=5) & S7A(3), Q9R, T10W, R11F, Q28R, V48I(2), V51A, K62R, A66G(3), P67A, T72N, P86Q/A(3), K87A(3), \\
& Q89P, R117C, V132I(2), F147S/A(4), A150V, I153L, V170I(3), N174S(2) \\
$3 \mathrm{a}(\mathrm{N}=9)$ & T16A, N27D, T30A, S37Y, A39T(3), I52M(9), A67V(3), P89S, A98T(6), S102A(9), V107I, L132I(2), \\
& A133S, A151V, S166A(8), V170I(8), T177S , A179T \\
\hline
\end{tabular}

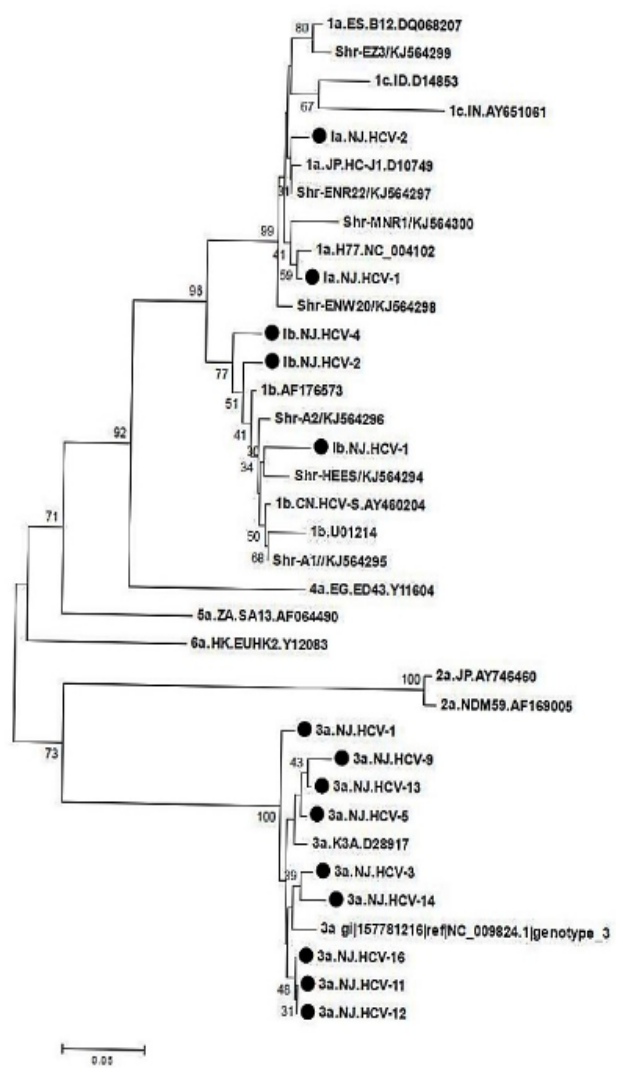

Figure 2. The Phylogenetic Analysis of 14 Samples based on NS3 Protease Region. All the samples including two $1 \mathrm{a}$, three $1 \mathrm{~b}$ and nine $3 \mathrm{a}$ sub-genotypes were correctly distributed into relevant clusters of tree by the help of neighbor-joining method. The investigated samples were highlighted by black circles in the constructed tree. The values on internal nodes are showing the percentage of reliability, bootstrapping value.

240 bp length was demonstrative of the virus presence in all samples after electrophoresis analysis. These 14 positive samples were included in the next step of analysis in protease sequence.

\section{Protease region amplification}

The new designed Nested PCR for amplification of NS3 protease showed suitable results in three different genotypes, $1 a, 1 b$ and $3 a$. The electrophoresis analysis demonstrated that for genotypes $1 a, 1 b$, and $3 a$ different bands with 700, 600 and 600 bp lengths were detectable, respectively as demonstrated in Figure 1.

\section{The Cloning Results}

The colony PCR assay was performed to ensure that the insertion of amplified protease region targets into plasmids. Regarding these results, the majority of white colonies were harboring recombined positive plasmids in spite of the ones with the blue color. Hence, out of 5 positive colonies that have been submitted for sequencing, 3 sequencing results were reliable and useful.

Sequence analysis showed a low frequency of PIs potential resistance substitution

The substitutions in amino acids sequence showed a relative conserve property in this region. However, some uncharacterized variations and few mutations responsible for potential resistance but not complete resistance was identified following the sequence analysis, as depicted in Figure 3 and Table 2

Among mutations with PIs resistance importance, only 1 out of 14 samples $(7 \%)$ harbored $R 117 C+N 174 S$ double mutation, which would causes reduced susceptibility to Telaprevir. It belong to a genotype 1a infected patient. No sign of other resistance mutation was detected among samples.

The most frequent substitutions were determined as $\operatorname{I52M}(N=9), S 102 A(N=9), S 166 A(8)$ and V170I (8) for genotype $3 a$, and $F 147 S / A(4)$ for genotype 1. However, some uncharacterized substitutions on scored position, including $1132 \mathrm{~L}(\mathrm{~N}=1), \operatorname{I170V}(\mathrm{N}=3)$ and $\mathrm{N174S}(\mathrm{N}=2)$ were also determined by Geno2Pheno assessment. Different types of variation were also detected by geno2pheno survey for genotype 1, among them $S 7 A(3)$, A66G (3), P86Q/A (3), K87A (3), V170I (3), were more prevalent. As well, in genotype 3 a mutations including $A 98 T$ (6), A39T (3) and A67V (3) were detected, as shown in Table 2.

\section{Phylogenetic Analysis}

The analysis of phylogenetic tree demonstrated a parallel result with nested-PCR findings. The constructed phylogenetic tree demonstrated that the sequence of each patient was correctly located in expected branch as belonged to relevant genotype. This finding showed that out of 14 samples, 2, 3 and 9 samples belonged to sub-genotypes $1 \mathrm{a}, 1 \mathrm{~b}$ and $3 \mathrm{a}$, respectively as shown in Figure 2. These sequences were submitted to NCBI GenBank with accession numbers: MK609550-MK609563. The finding highlights the power of NS3 protease region for construction of a valid phylogenetic tree and further genetic analysis of virus.

\section{Discussion}

Despite the availability of DAAs drugs for the treatment of $\mathrm{HCV}$, natural mutation still remains a big challenge (Vermehren and Sarrazin, 2011; Paolucci et al., 2013; Martinez et al., 2017). The rate of PIs natural mutations reported being wide to conclude for all areas 


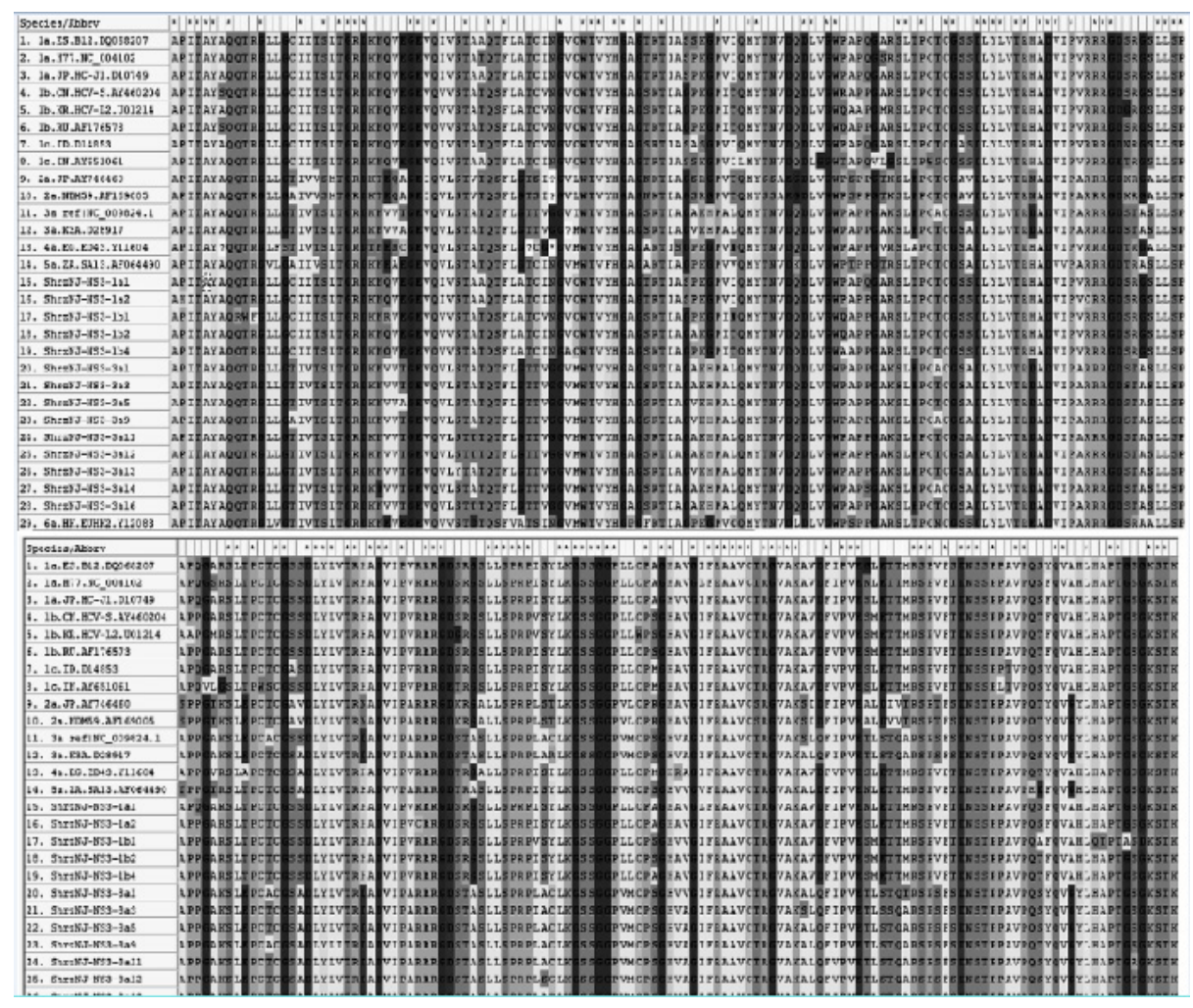

Figure 3. The Multiple Sequences Alignment of All Investigated Samples. The multiple alignment of new sequences with reference Hepatitis $\mathrm{C}$ virus sequences revealed different uncharacterized mutations and few substitutions responsible for PIs drug resistance. There are some other mutations that discussed in the text. Samples are nominated as ShrNJ-NS3 with a suffix of sample number.

and genotypes (Vallet et al., 2005; Lopez-Labrador et al., 2008; Paolucci et al., 2012; Martinez et al., 2017; Morsica et al., 2017). Although there are a couple of reports regarding this issue around the world, in Iran limited publications have considered it.

Actually, the impact of some PIs resistance on $\mathrm{HCV}$ $D A A$ s treatment should be considered as their occurrence before the starting of therapy may render resistance even when being treated with other DAAs (Paolucci et al., 2012; Lamb, 2017; Martinez et al., 2017; Morsica et al., 2017). Herein, NS3 protease sequence of a small patient population that determined as non-responder to Peg-IFN plus Ribavirin treatment were analyzed. This was the second study that had been done in Iran and the results demonstrated a similar finding to a previous report on a DAAs treatment-naive population (Afrasiabi et al., 2015).

Herein, variations responsible for PIs resistance were investigated by sequencing assay. From characterized mutations, only one out of 14 samples $(7 \%)$ harbored $R 117 C+N 174 S$ double mutation, which could render it a reduced susceptibility phenotype to Telaprevir. However, no PIs resistance related substitution was detected among samples. The importance of this type of mutation and the effect on resistance to PIs has been mentioned by different studies (Patino-Galindo et al 2016, Paolucci et al., 2012). This type of mutation should be considered in a screening method before the start of PIs therapy for patients.

Besides, there were some uncharacterized mutations in samples including V170I (11 out of 14) and I132L (4 out of 14$)$ and $N 174 S(N=2)$ in both genotype 1 and 3. Some of these variations were determined as "uncharacterized substitutions on scored position" by Geno2pheno software, due to their possible but not immediate role in PIs resistance(Kalaghatagi et al,. 2016). These kinds of mutations may render partial resistant phenotype to some extent against different PIs drugs (Paolucci et al., 2012, Patino-Galindo et al 2016). Similarly, these mutations have been detected in association with major resistant mutations in some other studies, however, their exact role on resistance phenotype and virus fitness needs to be evaluated (Sargin Altunok et al., 2016; Zhang et al., 2016).

A couple of experiments reported the different rate of heterogeneity and pre-existing substitutions with the potential but of PIs resistance (Kuntzen et al., 2008; Lopez-Labrador et al., 2008; Vallet et al., 2011; Paolucci et al., 2012; Morsica et al., 2017). In one clonal sequencing based investigation, variation in the protease region of 17 patients with genotype-1 showed a noticeable amount of heterogeneity alongside the protease region (Vallet et al., 2005). Kuntzen et al., (2008) following sequence analysis the finding of a high frequency of PIs resistance mutations (near 8\%) recommended a screening method of PIs resistance mutations before DAAs administration to reduce the cost and treatment failures. This data was supported by other regions such as Argentina (Jimenez-Sousa et al., 2016) even by the employment of traditional sequencing. The limited studies conducted in this regard in Iran indicated the presence of a critical $R 155 \mathrm{~K}$ mutation among a very small population (1 out of 7) (Afrasiabi et al., 2015). However, there are some reports claiming the low frequency of major PIS resistance mutations in an infected population. Different 
reports from Europe like ones by Vallet and others detected a low frequency of PIs critical mutations by direct sequencing (Vallet et al., 2011; Jimenez-Sousa et al., 2016).

In addition, the most prevalent substitutions were determined as $152 M(N=9), S 102 A(N=9)$ and S166A (8) for genotype $3 a$, and $F 147 S / A$ (4) for genotype 1. Whereas these kinds of polymorphisms were detected prevalently in other reports, their exact impacts on the protein function, virus fitness, and even drug susceptibility have to be defined (Paolucci et al., 2012).

The genotype determination of HCV confirmed to be reliable particularly when evaluated by analysis of NS5A gene (Dehghani-Dehej et al., 2017). However, other regions such as NS3 as an alternative candidate were taken into account by some other groups. As a candidate gene for genotyping purpose, it even showed a comparable sensitivity and specificity level with commercially available Versant HCV 2.0 reverse hybridization lineprobe assay (LiPA 2.0) for subtyping assay (Neukam et al., 2017). In our experiment, it was also revealed that NS3 protease region exhibits a reliable discriminative potential to differentiate between sub-genotype $1 a$, $1 b$, and $3 a$. The NS3-based phylogenetic tree and investigation in Geno2Pheno system truly revealed different sub-genotypes at relevant clusters, indicating the power of NS3 to molecularly differentiate among them.

The present study has some limitations, including the small size of population, heterogeneous genotype distribution among enrolled patients and a few numbers of investigated clones for further analysis.

In conclusion, with the exception of $R 117 C+N 174 S$ double mutation, confer a reduced susceptibility for Telaprevir, no sign of resistance mutation was found. Therefore, the low frequency of $P I S$ resistance mutations is hopeful to start these medications for IFN/RIB non-responder patients. However, despite the relevant reports regarding the low frequency of PIs resistance, the cost and side effects should inspire us to develop simple methods of resistance screening for treatment failure prevention. In addition, the NS3 protease region could serve as a reliable region for construction of a phylogenetic tree.

\section{Acknowledgments}

The present study was extracted from the thesis written by Narges Nejabat, which was financially supported by Shiraz University of Medical Sciences (Grant No. 94-9302). Special thanks to Zahra Mansourabadi and the staff of Liver Clinic, Gastroenterohepatology Research Center for their assistance.

\section{Conflict of interest}

All the authors declared no conflict of interest.

\section{References}

Afrasiabi M, Hosseini SY, Yaghobi R, et al (2015). Analysis of naturally occurring resistant mutations to Hepatitis $\mathrm{C}$ virus NS3 protease inhibitors: A preliminary study in south of
Iran. Jundishapur J Microbiol, 8, e24965.

Bartels DJ, Zhou Y, Zhang EZ, et al (2008). Natural prevalence of hepatitis $\mathrm{C}$ virus variants with decreased sensitivity to NS3 $\hat{A} \bullet 4 A$ protease inhibitors in treatment-naive subjects. J Infect Dis, 198, 800-7.

Courcambeck J, Bouzidi M, Perbost R, et al (2006). Resistance of hepatitis C virus to NS3-4A protease inhibitors: mechanisms of drug resistance induced by R155Q, A156T, D168A and D168V mutations. Antivir Ther, 11, 847-55.

Dehghani-Dehej F, Sarvari J, Esghaei M, et al (2017). Presence of different Hepatitis $\mathrm{C}$ virus genotypes in plasma and peripheral blood mononuclear cell samples of Iranian patients with HIV infection. J Med Virol, 90, 1343-51.

Jimenez-Sousa MA, Gutierrez-Rivas M, Alvaro-Meca A, et al (2016). NS3 Resistance-Associated Variants (RAVs) in patients infected with HCV genotype 1a in Spain. PLoS One, 11, e0163197.

Jindal G, Mondal D, Warshel A (2017). Exploring the drug resistance of HCV protease. $J$ Phys Chem B, 121, 6831-40.

Kalaghatgi P, Sikorski AM, Knops E, et al (2016). Geno2pheno[HCV]-A web-based interpretation system to support Hepatitis $\mathrm{C}$ treatment decisions in the Era of direct-acting antiviral agents. PLoS One, 11, e0155869.

Khanizadeh S, Ravanshad M, Hosseini SY, et al (2016). The possible role of NS3 protease activity of hepatitis $\mathrm{C}$ virus on fibrogenesis and miR-122 expression in hepatic stellate cells. Acta Virol, 60, 242-8.

Khanlari Z, Sabahi F, Hosseini SY, et al (2014). HCV NS3 blocking effect on IFN induced ISGs like Viperin and IL28 with and without NS4A. Hepat Mon, 14, e17822.

Khodabandehloo M, Roshani D (2014). Prevalence of hepatitis $\mathrm{C}$ virus genotypes in Iranian patients: a systematic review and meta-analysis. Hepat Mon, 14, e22915.

Kuntzen T, Timm J, Berical A, et al (2008). Naturally occurring dominant resistance mutations to hepatitis $\mathrm{C}$ virus protease and polymerase inhibitors in treatment native patients. Hepatology, 48, 1769-78.

Lamb YN (2017). Glecaprevir/Pibrentasvir: First Global Approval. Drugs, 77, 1797-1804.

Lopez-Labrador FX, Moya A, Gonzalez-Candelas F (2008). Mapping natural polymorphisms of hepatitis C virus NS3/4A protease and antiviral resistance to inhibitors in worldwide isolates. Antivir Ther, 13, 481-94.

MartinezAP, Culasso ACA, Perez PS, et al (2017). Polymorphisms associated with resistance to protease inhibitors in naive patients infected with hepatitis $\mathrm{C}$ virus genotype 1 in Argentina: Low prevalence of Q80K. Virus Res, 240, 140-6.

Morsica G, Andolina A, Merli M, et al (2017). NS3 protease resistance-associated substitutions in liver tissue and plasma samples from patients infected by hepatitis $\mathrm{C}$ virus genotype 1A or 1B. Arch Virol, 162, 2271-7.

Neukam K, Martinez AP, Culasso ACA, et al (2017). NS3 genomic sequencing and phylogenetic analysis as alternative to a commercially available assay to reliably determine hepatitis C virus subtypes $1 \mathrm{a}$ and $1 \mathrm{~b}$. PLoS One, 12, e0182193.

Paolucci S, Fiorina L, Mariani B, et al (2013). Naturally occurring resistance mutations to inhibitors of HCV NS5A region and NS5B polymerase in DAA treatment-naive patients. Virol J, 10, 355 .

Paolucci S, Fiorina L, Piralla A, et al (2012). Naturally occurring mutations to $\mathrm{HCV}$ protease inhibitors in treatment-naive patients. Virol J, 9, 245.

Patino-Galindo JA, Salvatierra K, González-Candelas F, et al., (2016). Comprehensive screening for naturally occurring Hepatitis $\mathrm{C}$ virus resistance to direct-acting antivirals in the $N S 3, N S 5 A$, and NS5B Genes in Worldwide Isolates of Viral 
Genotypes 1 to 6. Antimicrob Agents Chemother, 60, 2402-6.

Petruzziello A, Marigliano S, Loquercio G, et al (2016). Global epidemiology of hepatitis $C$ virus infection: An up-date of the distribution and circulation of hepatitis $\mathrm{C}$ virus genotypes. World J Gastroenterol, 22, 7824-40.

Romano KP, Ali A, Royer WE, et al (2010). Drug resistance against HCV NS3/4A inhibitors is defined by the balance of substrate recognition versus inhibitor binding. Proc Natl Acad Sci U S A, 107, 20986-91.

Sargin Altunok E, Sayan M, Akhan S, et al (2016). Protease inhibitors drug resistance mutations in Turkish patients with chronic Hepatitis C. Int J Infect Dis, 50, 1-5.

Vallet S, Gouriou S, Nousbaum JB, et al (2005). Genetic heterogeneity of the NS3 protease gene in hepatitis $\mathrm{C}$ virus genotype 1 from untreated infected patients. J Med Virol, 75, 528-37.

Vallet S, Viron F, Henquell C, et al (2011). NS3 protease polymorphism and natural resistance to protease inhibitors in French patients infected with HCV genotypes 1-5. Antivir Ther, 16, 1093-102.

Vermehren J, Sarrazin C (2011). New HCV therapies on the horizon. Clin Microbiol Infect, 17, 122-34.

Vicenti I, Rosi A, Saladini F, et al (2012). Naturally occurring hepatitis $\mathrm{C}$ virus (HCV) NS3/4A protease inhibitor resistance-related mutations in $\mathrm{HCV}$ genotype 1-infected subjects in Italy. J Antimicrob Chemother, 67, 984-7.

Zeminian LB, Padovani JL, Corvino SM, et al (2013). Variability and resistance mutations in the hepatitis $\mathrm{C}$ virus NS3 protease in patients not treated with protease inhibitors. Mem Inst Oswaldo Cruz, 108, 13-7.

Zhang Y, Cao Y, Zhang R, et al (2016). Pre-Existing HCV variants resistant to DAAs and their sensitivity to PegIFN/ RBV in Chinese HCV genotype $1 \mathrm{~b}$ patients. PLoS One, 11, e0165658.

\section{(c) (i) (8)}

This work is licensed under a Creative Commons AttributionNon Commercial 4.0 International License. 